\title{
O047: The effect of active surveillance culture of carbapenem resistant Acinetobacter baumannii on the occurrence of carbapenem resistant Acinetobacter baumannii bacteremia in a single intensive care unit
}

\author{
$\mathrm{G} \mathrm{Kim}^{1 *}, \mathrm{~B} \mathrm{Oh}^{2}$, JS Song ${ }^{2}$ PG Choe ${ }^{3}$, WB Park ${ }^{3}, \mathrm{HB} \mathrm{Kim}{ }^{4}$, N-J Kim³ ${ }^{3}, \mathrm{EC} \mathrm{Kim}{ }^{5}, \mathrm{M}-\mathrm{D} \mathrm{Oh}^{3}$
}

From 2nd International Conference on Prevention and Infection Control (ICPIC 2013)

Geneva, Switzerland. 25-28 June 2013

\section{Introduction}

Carbapenem resistant Acinetobacter baumannii (CRAB) is an emerging pathogen of healthcare associated infection and little is known about the effectiveness of the active surveillance culture of CRAB.

\section{Objectives}

This study aims at evaluating the effect of active surveillance culture of CRAB upon intensive care unit(ICU) admission on the occurrence of new CRAB bacteremia in ICU.

\section{Methods}

Since February 2011, the active surveillance culture of CRAB was performed in all patients admitted to medical ICU in Seoul national university hospital. Contact precaution was applied for the patients who had positive surveillance culture results. Respiratory specimen were obtained and the isolates were cultured overnight in blood agar plates which contain imipenem. To assess the effectiveness of active surveillance culture of CRAB and contact precaution, the rate of new CRAB bacteremia was compared between baseline period (February 2012-January 2011) and intervention period (June 2011February 2012). The new CRAB bacteremia case was defined as the CRAB bacteremia occurred later than 48 hours of ICU admission in patients without positive

${ }^{1}$ Internal Medicine, National Medical Center, Seoul National University Hospital, Seoul, Korea, Republic Of

Full list of author information is available at the end of the article
CRAB clinical culture in the prior 12 months and within the 48 hours of ICU admission.

\section{Results}

In the baseline period there were 242 total admissions to the ICU (7355 patients-days) and in the intervention period there were 266 total admissions to the ICU (5432 patients-days). During the intervention period, 21(7.9\%) patients showed positive CRAB surveillance culture results. The rate of new CRAB bacteremia is 2.72 cases per 1000 patients-days in the baseline period and 0.92 case per 1000 patients-days in the intervention period $(P=0.019)$.

\section{Conclusion}

When active surveillance culture of CRAB and contact precaution for the patients of positive results were applied in a medical ICU, the rate of new CRAB bacteremia was lowered and the time betweennew CRAB bacteremia and ICU admission was lengthened.

\section{Disclosure of interest}

None declared.

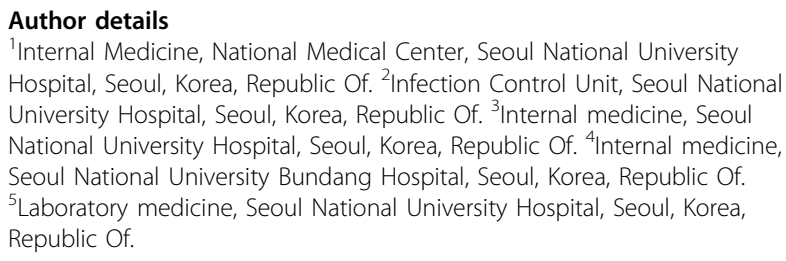


Cite this article as: Kim et al: O047: The effect of active surveillance culture of carbapenem resistant Acinetobacter baumannii on the occurrence of carbapenem resistant Acinetobacter baumannii bacteremia in a single intensive care unit. Antimicrobial Resistance and Infection Control 2013 2(Suppl 1):047.

Submit your next manuscript to BioMed Central and take full advantage of:

- Convenient online submission

- Thorough peer review

- No space constraints or color figure charges

- Immediate publication on acceptance

- Inclusion in PubMed, CAS, Scopus and Google Scholar

- Research which is freely available for redistribution

Submit your manuscript at www.biomedcentral.com/submit
C Biomed Central 